\title{
Modern hip fracture care still involves nearly 3 days of bed rest - findings of the national Physiotherapy 'Hip Sprint' Audit in 2017
}

\author{
Authors: Elizabeth Fagan, ${ }^{\mathrm{A}}$ James Hannaford, ${ }^{\mathrm{A}}$ Meghan Liddicoat, ${ }^{\mathrm{A}}$ Ruth ten Hove ${ }^{\mathrm{B}}$ and Antony Johansen ${ }^{\mathrm{A}}$
}

\section{Introduction}

Modern anaesthesia and surgery are now so successful that nearly all patients with hip fracture receive prompt effective repair of their injury. This condition is an excellent test of the challenges faced by all frail and older hospital inpatients and by the multidisciplinary teams who seek to restore their previous mobility, independence and quality of life.

\section{Methodology}

The National Hip Fracture Database (NHFD) at the Royal College of Physicians collects data on all patients presenting with hip fracture in England and Wales. In May-June 2017 the Chartered Society of Physiotherapy led work by over 580 physiotherapists; supplementing NHFD data with detailed information about the rehabilitation provided to $5,989(78.6 \%)$ of the 7,621 people who presented to 127 participating hospitals.

\section{Results}

On average each patient received 2 hours of physiotherapy (118 minutes) in the week after operation. $68.4 \%$ were mobilised out of bed by the day following operation. When added to the time spent waiting for their operation this meant that a typical patient still faces nearly 3 days of bed rest ( 2.66 days) before they can get out of bed after hip fracture. The 'Hip Sprint' Audit found significant variation in performance; nine hospitals (7\%) achieved this for fewer than half of patients. $9.4 \%$ of patients were unable to get up as a result of pain or hypotension; factors which might have been anticipated and avoided by clear perioperative protocols and closer working with surgical and anaesthetic colleagues.

\section{Discussion}

Patients should be helped to get up as soon as possible after surgery - this is key to their wellbeing and avoidance of complications such as delirium. Collaborative multidisciplinary working is needed to ensure that pain, hypotension and delirium

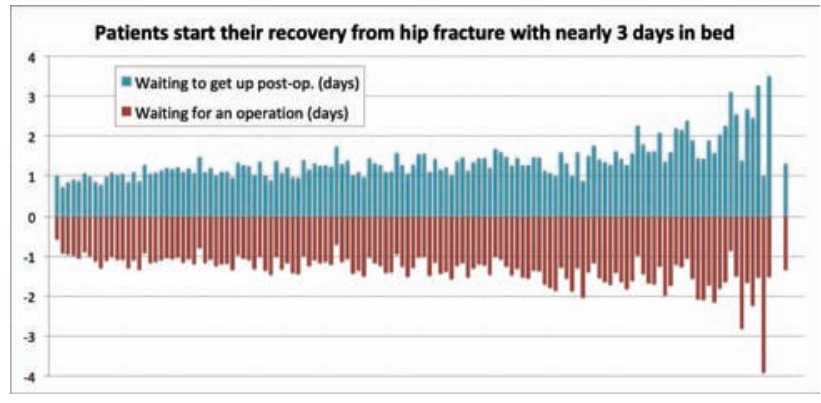

Fig 1. Patients start their hip fracture recovery with nearly 3 days in bed

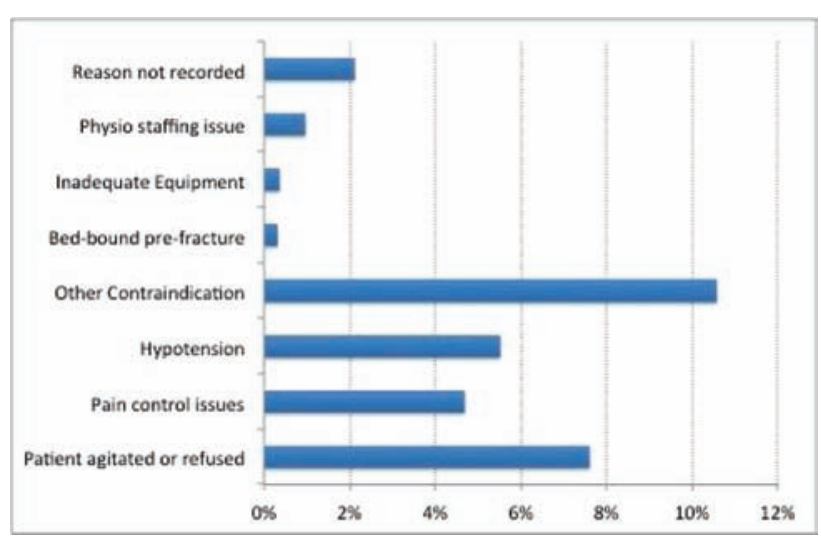

Fig 2. Reasons for being unable to get up

do not delay the start of rehabilitation. Individual teams should review the picture of their hospital's immediate postoperative management provided at www.fffap.org.uk/phfsa.

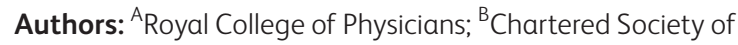
Physiotherapy 